九州大学学術情報リポジトリ

Kyushu University Institutional Repository

\title{
Reaction of 2-Amino-5-Substituted-l, 3, 4- Thiadiazole with 1, 3-Dicarbonyl Compound
}

Okabe, Takayuki

Laboratory of Pesticide Chemistry, Faculty of Agriculture, Kyushu University

Taniguchi, Eiji

Laboratory of Pesticide Chemistry, Faculty of Agriculture, Kyushu University

Maekawa, Kazuyuki

Laboratory of Pesticide Chemistry, Faculty of Agriculture, Kyushu University

https://doi.org/10.5109/22831

出版情報: 九州大学大学院農学研究院紀要. 17 (2)，pp. 195-202，1973-03. Kyushu University バージョン：

権利関係 : 


\title{
Reaction of 2-Amino-5-Substituted-1, 3, 4-Thiadiazole with 1,3-Dicarbonyl Compound
}

\author{
Takayuki Okabe, Eiji Taniguchi and Kazuyuki Maekawa \\ Laboratory of Pesticide Chemistry, Faculty of Agriculture, \\ Kyushu University, Fukuoka
}

(Received October 12, 1972)

\begin{abstract}
The synthesis of thiadiazolopyrimidines by heating a-amino-1, 3,4-thiadiazoles with 1,3-dicarbonyl compounds in polyphosphoric acid has been investigated. The products obtained from 2 -amino-l, 3, 4-thiadiazoles with $\beta$-keto-esters were $5 H$-thiadiazolo[3,2a]pyrimidin-5-ones, and those obtained from 2-amino-1,3,4-thiadiazoles with 1, 3-dicarbony1 compounds were thiadiazolo $[3,2-a]$ pyrimidin-4-ium compounds. It was also found that the reaction of 2-amino-5-mercapto-1,3,4-thiadiazole with alkyl acetoacetate yielded 7-methyl-2-a1kylthio-5 $\boldsymbol{H}$-thiadiadiazolo[3,2-a] pyrimidin-5-one.
\end{abstract}

Present paper deals with the synthesis of thiadiazolopyimidines (II, III) by a condensation reaction of 2 -amino-5-substituted-1, 3, 4-thiadiazoles (I) with 1,3dicarbonyl compounds such as $\beta$-keto-esters and 1, 3-diketones.

First, the condensing ring closure reaction was tried according to Allen et al. (1959), who synthesized $5 H$-thiadiazolo [3,2-a] pyrimidin-5-ones by heating 2-aminothiadiazoles with ethyl acetoacetate or diethyl ethoxymethylenemalonate in trichlorobenzene (TCB).

Except ethyl acetoacetate and ethoxymethylenemalonate, $\beta$-keto-esters did not give thiadiazolopyrimidines, but acylaminothiadiazoles, e. g., ethyl benzoylacetate and diethyl malonate gave 2-benzoylacetamino-1,3,4-thiadiazole and $\mathrm{N}$, $\mathrm{N}^{\prime}$-dithiadiazoyl malonamide, respectively.

The ring closure reaction in alkaline media (soduim alcoholate, and NaHdimethyl sulfoxide) was found to be unsuccessful, and only intermediary amides were isolated ; e. g., acetoacetate reacted with 2-aminothiadiazole to 2-acetoacetylamino-1,3,4-thiadiazole (IV).

In polyphosphoric acid (PPA), the condensation reaction to thiadiazolopyrimidine satisfactorily proceeded, using $\beta$-keto-ester such as acetoacetate, $\beta$ chloroacetoacetate, benzoylacetate (Bowden and Brown, 1971; Singh et al., 1970). However, diethyl malonate, diethyl 2-methylmalonate and ethyl cyanoacetate gave again 2-acylaminothiadiazoles (VII, VIII).

1,3-Diketone (acetylacetone) or dialdehyde (1,1,3,3-tetraethoxypropane (Pollak et al., 1971)) condensed with 2-aminothiadiazole in PPA to yield thiadiazolo [3,2-a] pyrimidin-4-ium compounds (III). The synthetic route is shown in Fig. 1.

On the basis of IR and UV absorption spectra, the thiadiazolopyrimidines 


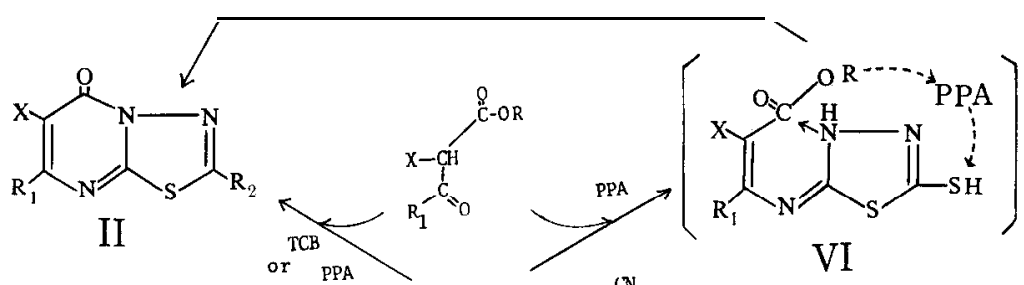

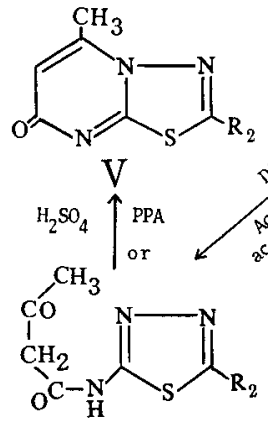

IV<smiles></smiles>

III
VI

${ }_{4}^{(N)}-C_{2}$<smiles>[R]c1nnc(NC(C)=O)s1</smiles>

VII

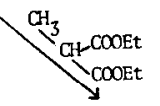<smiles>[R2]c1nnc(NC(C)C)s1</smiles>

VIII

Fig. 1. Reaction of 2-amino-1,3,4-thiadiazoles and $\beta$-keto-esters.

synthesized in PPA were assigned to $5 H$-thiadiazolo[3, 2-a] pyrimidin-5-ones (II), but not to the isomer 7-ones (V) which were derived from 2-acetoacetylamino-1, 3, 4-thiadiazoles (IV).

The absorption spectra of II and V showed a significant difference. The

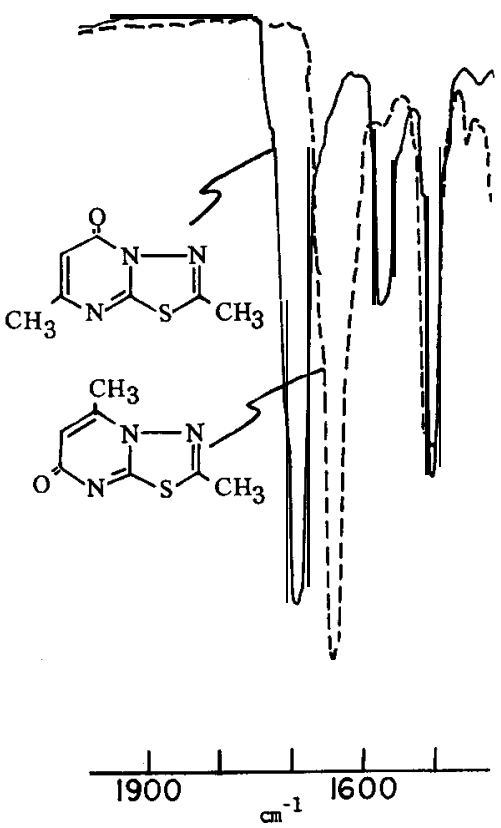

Fig. 2. IR-spectra of 2,7-dimethyl-5H-thiadiazolo[3,2-a]pyrimidin-5-one and 2,5-dimethyl-7 $H$-thiadiazolo $[3,2-a]$ py rimidin-7-one. 
amido I absorption of the 7-one appears below $1650 \mathrm{~cm}^{-6}$, while that of the 5one is above $1690 \mathrm{~cm}^{-1}$ (Fig. 2). The main UV absorption band of the 7-one is in much shorter wavelength than that of the 5-one (Fig. 3). A similar spectral difference between thiazolopyrmidin-5-ones and 7-ones has been reported by Dunwell and Evans in 1971.

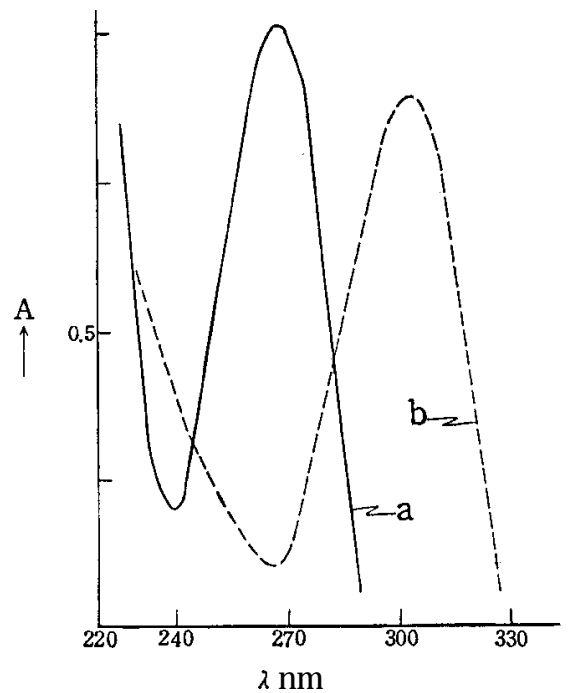

Fig. 3. UV-spectra of 2,7-dimethyl-5H-thiadiazolo[3,2-a]pyrimidin-5-one and 2,5-dimethyl-7 $H$-thiadiazolo[3,2-a]pyrimidin-7-one.

a (-) 7-one compound, b (-.-) 5-one compound

Incidentally, 2-amino-5-mercapto-1, 3, 4-thiadiazole (I. $\mathrm{R}=\mathrm{SH}$ ) condensed with methyl (or ethyl) acetoacetate to give 2-methylthio (or 2-ethylthio)-7-methyl-5Hthiadiazolo [3, 2-a] pyrimidin-5-one (II, $\mathrm{X}=\mathrm{CH}_{3}, \mathrm{R}=\mathrm{SCH}_{3}$ or $\mathrm{SC}_{2} \mathrm{H}_{6}$ ). These compounds were identical on IR and mixed melting point with those synthesized from 2-amino-5-methylthio (or ethylthio)-1, 3, 4-thiadiazole (I, $\mathrm{R}=\mathrm{SCH}_{3}$ or $\mathrm{SC}_{2} \mathrm{H}_{5}$ ) and ethyl acetoacetate. Therefore, it is evident that the alkyl of alkylmercapto group in the product originated from the alkyl group of the ester (Fig. 4).

\section{EXPERIMENTAL}

\section{1) 7-Methyl-5H-thiadiazolo $[3,2-a]$ pyrimidind-ones (II, $\mathrm{R}_{\mathbf{1}}=\mathrm{Me}, \mathrm{X}=\mathrm{H}$ )}

These compounds were prepared by following methods. Details of the products are enumerated in Table 1.

Method A : A mixture of 2-amino-1, 3, 4-thiadiazoles (0.015 mole) (Chubb and Nissenbaum, 1959), ethyl acetoacetate $(0.017 \mathrm{~mole})$, and TCB $(25 \mathrm{ml})$ was heated at $150-160^{\circ}$ for about 5 hours under reduced pressure (Allen et al., 1959 ; Kuderna et al., 1971). The cooled mixture was diluted with pet. ether, and then resulting crystals were recrystallized from AcOEt.

Method B: A mixture of I (0.02 mole), and ethyl acetoacetate (0.02 mole) in 


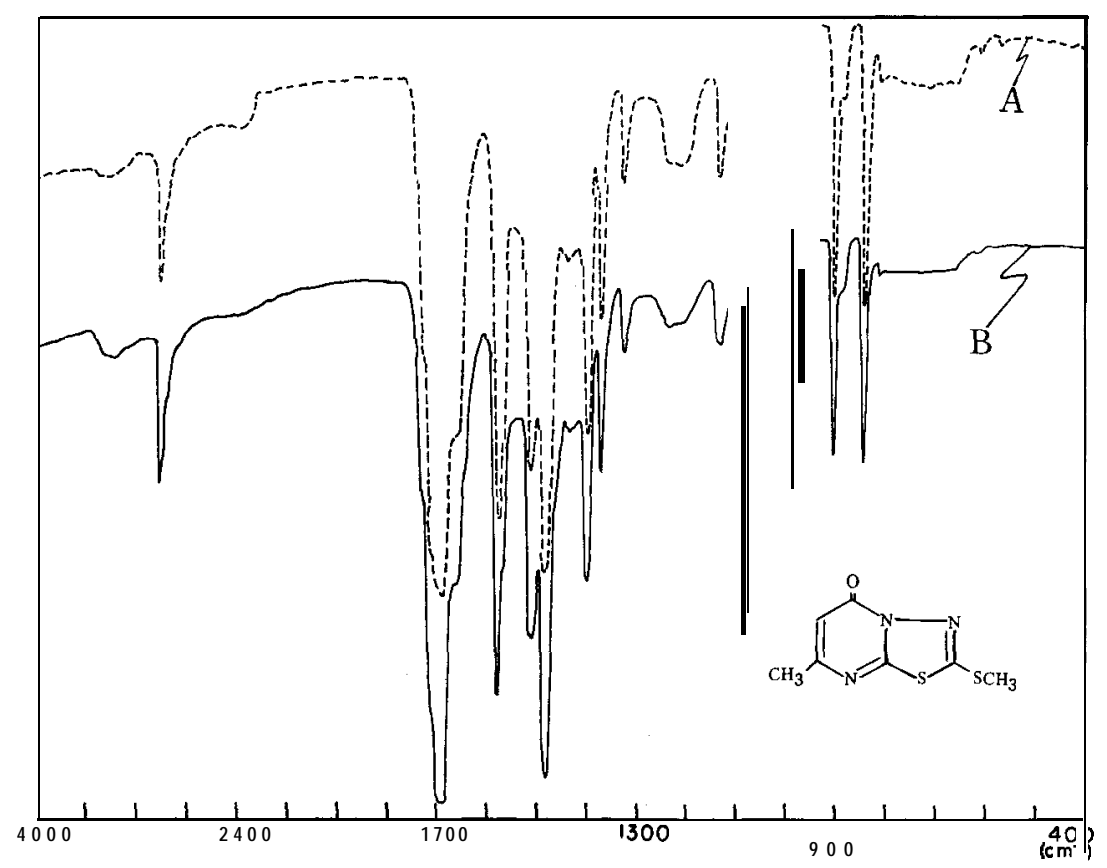

Fig. 4. IR-spectra of 7-methyl-2-methylthio-5H-thiadiazolo[3,2-a]pyrimidin-5-one, A : Authentic compound. B : The compound obtained from a-amino5-mercapto-1,3,4-thiadiazole by the alkyltransfer.

PPA (15 g) was heated at $120-160$ " for $30-90$ min. The cooled mixture was diluted with water and extracted with $\mathrm{CHCl}_{3}$. The solvent was removed under reduced pressure from the dried (over $\mathrm{Na}_{2} \mathrm{SO}_{4}$ ) extract, and the residue was recrystallized from $\mathrm{CHCl}_{3}$-AcOEt (1:3) to give colorless needles.

2) 2,7-Dimethyl-6-chloro-5 $\boldsymbol{H}$-thiadiazolo [3,2-u] pyrimidin-S-one (II, $\mathbf{R}_{1}, \mathbf{R}_{2}=\mathrm{Me}$, $\mathbf{X}=\mathbf{C l})$

A suspension of an equimolecular amount of I and ethyl $\beta$-chloroacetoacetate in PPA was heated at 140-160" for 20-60 min. Then, the cooled mixture was poured into ice-water, resulting crystals were recrystallized from AcOEt-MeOH (3: 1) to give colorless needles. Yield, $84 \%$.

3) 2-Methylthio-7-methyl-5 $H$-thiadiazolo $[3,2-a]$ pyrimidin-S-one, (II, $\mathbf{R}_{\mathbf{1}}=\mathbf{M e}, \mathbf{R}_{2}$ $=\mathrm{S}-\mathrm{Me}, \quad \mathrm{X}=\mathbf{H}$ )

A mixture of 2-amino-5-mercapto-1, 3, 4-thiadiazole $(2.7 \mathrm{~g})$, methyl acetoacetate $(2.5 \mathrm{~g})$ and PPA $(10 \mathrm{~g})$ was heated at $140-150$ " for $30 \mathrm{~min}$. The cooled mixture was poured into ice-water, and extracted with chloroform. The dried extract was concentrated under reduced pressure to get yellow residue, which was recrystallized from EtOH to get colorless needles $(2.8 \mathrm{~g}, 67 \%), \mathrm{m}$. p. $154^{\circ} \nu_{\max },\left(\mathrm{cm}^{-1}\right)$ 1685. 
Table 1. Syntheses of thiadiazolo[ 3,2-a] pyrimidine derivatives.

\begin{tabular}{|c|c|c|c|c|c|c|c|c|c|c|c|c|c|}
\hline \multicolumn{3}{|c|}{ Formula II } & \multicolumn{3}{|c|}{ Reaction condition } & \multirow{2}{*}{$\begin{array}{l}\text { Yield } \\
(\%)\end{array}$} & \multirow{2}{*}{ M.p. } & \multirow{2}{*}{$\nu_{\mathrm{cm}^{-1}}$} & \multirow{2}{*}{$\begin{array}{l}\text { Molecular } \\
\text { formula }\end{array}$} & \multicolumn{3}{|c|}{ Analysis $(\%) \frac{\text { Calcd. }}{\text { Found }}$} & \multirow{2}{*}{ Ref. } \\
\hline $\mathrm{R}_{1}$ & $\mathrm{R}_{2}$ & $x$ & $\begin{array}{l}\text { Time } \\
(\mathrm{h} r .)\end{array}$ & $\begin{array}{l}\text { Temp. } \\
\text { (“C) }\end{array}$ & Solvent & & & & & C & $\mathrm{H}$ & $\mathrm{N}$ & \\
\hline $\mathrm{Me}$ & $\mathrm{Me}$ & $\mathrm{Cl}$ & 1 & 140 & PPA & 84 & 145 & 1690 & $\mathrm{C}_{7} \mathrm{H}_{6} \mathrm{ON}_{3} \mathrm{SCl}$ & $\begin{array}{l}38.99 \\
38.89\end{array}$ & $\begin{array}{l}2.81 \\
2.89\end{array}$ & $\begin{array}{l}19.49 \\
19.52\end{array}$ & \\
\hline $\mathrm{Me}_{3}$ & Et & $\mathrm{Cl}$ & 0.3 & $150-160$ & " & 29 & 150 & 1690 & $\mathrm{C}_{3} \mathrm{H}_{8} \mathrm{ON}_{3} \mathrm{SCl}$ & $\begin{array}{l}41.84 \\
41.77\end{array}$ & $\begin{array}{l}3.53 \\
3.51\end{array}$ & $\begin{array}{l}18.31 \\
18.39\end{array}$ & \\
\hline $\mathrm{Me}$ & $\mathrm{H}_{3}>\mathrm{Cl}$ & $\mathrm{Cl}$ & 0.5 & 155 & " & 68 & 155.5 & 1690 & $\mathrm{C}_{9} \mathrm{H}_{10} \mathrm{~N}_{3} \mathrm{SCl}$ & $\begin{array}{l}44.36 \\
44.57\end{array}$ & $\begin{array}{l}4.14 \\
4.26\end{array}$ & $\begin{array}{l}17.25 \\
17.44\end{array}$ & \\
\hline $\mathrm{Me}$ & $\mathrm{S}-\mathrm{Me}$ & $\mathrm{Cl}$ & 1 & 140 & " & 83 & 172 & 1695 & $\mathrm{C}_{7} \mathrm{H}_{6} \mathrm{ON}_{3} \mathrm{~S}_{2} \mathrm{Cl}$ & $\begin{array}{l}33.95 \\
33.87\end{array}$ & $\begin{array}{l}2.44 \\
2.51\end{array}$ & $\begin{array}{l}16.97 \\
16.87\end{array}$ & \\
\hline $\mathrm{H}$ & Et & $\mathrm{CO}_{2} \mathrm{Et}$ & 1 & reflux & TCB & 59 & 93 & 1720 & $\mathrm{C}_{10} \mathrm{H}_{11} \mathrm{ON}_{3} \mathrm{~S}$ & $\begin{array}{l}47.43 \\
47.33\end{array}$ & $\begin{array}{l}4.38 \\
4.33\end{array}$ & $\begin{array}{l}16.60 \\
16.81\end{array}$ & 5,6 \\
\hline $\mathrm{Me}$ & $\mathrm{Me}$ & $\mathrm{H}$ & 1.5 & $140-160$ & PPA & 84 & 154 & 1690 & $\mathrm{C}_{7} \mathrm{H}_{7} \mathrm{ON}_{3} \mathrm{~S}$ & $\begin{array}{l}46.41 \\
46.35\end{array}$ & $\begin{array}{l}3.90 \\
3.89\end{array}$ & $\begin{array}{l}23.20 \\
23.32\end{array}$ & \\
\hline $\mathrm{Me}$ & Et & $\mathrm{H}$ & 0.5 & $150-160$ & " & 90 & 112 & 1690 & $\mathrm{C}_{8} \mathrm{H}_{9} \mathrm{ON}_{3} \mathrm{~S}$ & $\begin{array}{l}49.23 \\
49.23\end{array}$ & $\begin{array}{l}4.65 \\
4.58\end{array}$ & $\begin{array}{l}21.53 \\
21.52\end{array}$ & 1 \\
\hline $\mathrm{Me}$ & $\left.: \mathrm{H}_{3}>\mathrm{C}\right]$ & $\mathrm{H}$ & 1 & $140-150$ & " & 50 & 79 & 1690 & $\mathrm{C}_{9} \mathrm{H}_{11} \mathrm{ON}_{3} \mathrm{~S}$ & $\begin{array}{l}51.67 \\
51.52\end{array}$ & $\begin{array}{l}5.30 \\
5.42\end{array}$ & $\begin{array}{l}20.09 \\
20.08\end{array}$ & \\
\hline$\phi$ & Et & $\mathrm{H}$ & 2 & $140-1.50$ & " & 34 & 144 & 1680 & $\mathrm{C}_{13} \mathrm{H}_{11} \mathrm{ON}_{3} \mathrm{~S}$ & $\begin{array}{l}60.69 \\
60.97\end{array}$ & $\begin{array}{l}4.31 \\
4.36\end{array}$ & $\begin{array}{l}16.34 \\
16.46\end{array}$ & \\
\hline
\end{tabular}


Anal. Calcd. for $\mathrm{C}_{7} \mathrm{H}_{7} \mathrm{ON}_{\mathrm{s}} \mathrm{S}_{2}$ : C, $39.44 \quad \mathrm{H}, 3.31 \quad \mathrm{~N}, 19.72$

Found: C, $39.59 \quad$ H, $3.27 \quad$ N, 19.79

This compound was also synthesized by heating an equimolar solution of 2 amino-5-methylthio-1, 3, 4-thiadiazole and ethyl acetoacetate in PPA. Yield, 62 $\%$, m. p. $154^{\circ}, \nu_{\max }\left(\mathrm{cm}^{-1}\right) 1685$.

Anal. Calcd. for $\mathrm{C}_{7} \mathrm{H}_{7} \mathrm{ON}_{3} \mathrm{~S}_{2}:$ C, $39.44 \quad \mathrm{H}, 3.31 \quad \mathrm{~N}, 19,72$

Found: C, $39.44 \quad \mathrm{H}, 3.27 \quad \mathrm{~N}, 19.79$

4) 2-Ethylthio-7-methyl-5 $H$-thiadiazolo $[3,2$-a $]$ pyrimidin-5-one (II, R, $=\mathbf{M e}, \mathbf{R}_{2}=\mathbf{S}$ Et, $X=H$ )

This compound was obtained from 2-amino-5-mercapto-1,3, 4-thiadiazole and ethyl acetoacetate in $70 \%$ yield, m. p. $119-119.5^{\circ}, \nu_{\max }\left(\mathrm{cm}^{-1}\right) 1698$.

Anal. Calcd. for $\mathrm{C}_{8} \mathrm{H}_{9} \mathrm{ON}_{3} \mathrm{~S}_{2}: \mathrm{C}, 42.29 \quad \mathrm{H}, 3.99 \quad \mathrm{~N}, 18.50$

Found : C, $42.26 \quad$ H, $3.96 \quad$ N, 13.52

The same compound was also obtained by heating 2-amino-5-ethylthio-1, 3, 4thiadiazole and ethyl acetoacetate in PPA in $97 \%$ yield, m. p. $119-120^{\circ}, \nu_{\max }\left(\mathrm{cm}^{\circ}\right)$ 1685 .

Anal. Calcd. for $\mathrm{C}_{8} \mathrm{H}_{9} \mathrm{ON}_{3} \mathrm{~S}_{2}: \mathrm{C}, 42.29 \mathrm{H}, 3.99 \quad \mathrm{~N}, 18.50$

Found : C, $42.52 \quad \mathrm{H}, 3.99 \quad \mathrm{~N}, 18.45$

5) 2-Methyl-7-phenyl-5 $H$-thiadiazolo $[3,2-a]$ pyrimidin-5-one (II, $\mathbf{R}_{1}=\mathbf{O}, \mathbf{R}_{2}=\mathrm{Me}$, $\mathbf{X}=\mathbf{H})$

A mixture of 2-amino-5-methyl-1,3, 4-thiadiazole (2.3 g), ethyl benzoylacetate (4 g), and PPA (15 g) was heated at 130-140" for $40 \mathrm{~min}$. The cooled mixture was diluted with water, and the resulting crystals were collected, washed with water, and recrystallized from $\mathrm{MeOH}$ as colorless needles. Yield $1.8 \mathrm{~g}, \mathrm{~m}$. p. 195$195.5^{\circ}, \nu_{\max }\left(\mathrm{cm}^{-}\right)$1690,

Anal. Calcd. for $\mathrm{C}_{12} \mathrm{H}_{9} \mathrm{ON}_{3} \mathrm{~S}: \mathrm{C}, 59.26 \mathrm{H}, 3.73 \quad \mathrm{~N}, 17.28$

Found: $\mathrm{C}, 59.33 \quad \mathrm{H}, 3.79 \quad \mathrm{~N}, 17.29$

\section{6) 2-Acetoacetylamino-1, 3, 4-thiadiazole (IV)}

Method C: Diketene (2.3 g) was added dropwise to a suspension of I ( $2.7 \mathrm{~g})$ in dried benzene $(20 \mathrm{ml})$. The mixture was heated under reflux for $60 \mathrm{~min}$. After cooling, the resulting crystals were recrystallized from $\mathrm{MeOH}$ as colorless needles $(4.3 \mathrm{~g})$, m. p. $178.5 \%$.

Anal. Calcd. for $\mathrm{C}_{6} \mathrm{H}_{7} \mathrm{O}_{2} \mathrm{~N}_{3} \mathrm{~S}: \mathrm{C}, 33.92 \mathrm{H}, 3.81, \quad \mathrm{~N}, 22.70$

Found : C, $39.08 \quad$ H, 3.73. N, 22.95

Method D: To a suspension of I $(5.5 \mathrm{~g})$ in absolute MeOH containing sodium methylate $(1 \mathrm{~g} \mathrm{Na}), 7.3 \mathrm{~g}$ of ethyl acetoacetate was added, and refluxed for 8 hours. The solvent was removed under reduced pressure and the oily residue was dissolved in water and filtered. The filtrate was acidified with conc. $\mathrm{HCl}$ to 
obtain a crude product. The product was recrystallized from $\mathrm{MeOH}$ to get colorless needles (3.5 g), m. p. 178.5”.
Anal. Calcd. for $\mathrm{C}_{6} \mathrm{H}_{7} \mathrm{O}_{2} \mathrm{~N}_{3} \mathrm{~S}: \mathrm{C}, 38.92$
$\mathrm{H}, 3.81 \quad \mathrm{~N}, 22.70$
Found: C, 38.97
$\mathrm{H}, 3.80$
$\mathrm{N}, 22.67$

\section{7) 2, 5-Dimethyl-7H-thiadiazolo [3,2-u] pyrimidii-'I-one (V, $\mathbf{R}_{2}=\mathbf{M}$ e)}

M ethod E: -(with conc. $\left.\mathrm{H}_{2} \mathrm{SO}_{4}\right)$ -

A suspension of 2-acetoacetylamino-5-methyl-1,3,4-thiadiazole $(2.3 \mathrm{~g})$ in conc. $\mathrm{H}_{2} \mathrm{SO}_{4}$ was heated at 50-60" for 5 hours. The cooled mixture was poured into ice-water, and neutralized with $\mathrm{Na}_{2} \mathrm{CO}_{3}$, then extracted with chloroform. The dried chloroform extract was concentrated under reduced pressure to get yellow solid residue. The residue was recrystallized from $\mathrm{MeOH}$-petroleum ether to get colorless needles $(1.0 \mathrm{~g}), \mathrm{m}$. p. $189-190^{\circ}, \nu_{\max }\left(\mathrm{cm}^{-1}\right) 1642$.

$\begin{array}{rrr}\text { Anal. Calcd. for } \mathrm{C}_{7} \mathrm{H}_{7} \mathrm{ON}_{3} \mathrm{~S}: \mathrm{C}, 46.41 & \mathrm{H}, 3.90 & \mathrm{~N}, 23.20 \\ \text { Found : C, } 46.83 & \mathrm{H}, 3.77 & \mathrm{~N}, 23.33\end{array}$

M ethod B: - (with PPA)-

A mixture of 2-acetoacetylamino-5-methyl-1,3,4-thiadiazole (4.8 g) and PPA (15 g) was heated at $140-150^{\circ}$ for $50 \mathrm{~min}$. The cooled mixture was diluted with water and neutralized with conc. $\mathrm{NH}_{4} \mathrm{OH}$. Upon standing at room temperature overnight, pale yellow crystals formed. The product was recrystallized from AcOEt-ether to get colorless needles $(800 \mathrm{mg}), \mathrm{m}$. p. $188-189.5^{\circ}, \nu_{\max }\left(\mathrm{cm}^{-1}\right) 1643$, $\mathrm{UV}(\mathrm{EtOH}) \lambda_{\max } 268 \mathrm{~nm}$.

Anal. Calcd. for $\mathrm{C}_{7} \mathrm{H}_{7} \mathrm{ON}_{3} \mathrm{~S}$ : C, $46.41 \quad \mathrm{H}, 3.90 \quad \mathrm{~N}, 23.20$

Found: C, $46.65 \quad$ H, $3.77 \quad$ N, 23.25

\section{8) 5-Methyl-7H-thiadiazolo [3,2-u] pyrimidin-7-one, $\left(\mathrm{V}, \mathbf{R}_{2}=\mathrm{H}\right)$}

This compound was obtained by heating 2-acetoacetylamino-1, 3, 4-thiadiazole in conc. $\mathrm{H}_{2} \mathrm{SO}_{4}$ at 50-60" for 2 hours according to the method E. Yield, $16 \%, \mathrm{~m}$. p. $194.5^{\circ}, \nu_{\max }\left(\mathrm{cm}^{-1}\right) 1645$, UV (EtOH), $\lambda_{\max } 268 \mathrm{~nm}$.

$\begin{array}{rrr}\text { Anal. Calcd. for } \mathrm{C}_{6} \mathrm{H}_{5} \mathrm{ON}_{3} \mathrm{~S}: \mathrm{C}, 43.12 & \mathrm{H}, 3.02 & \mathrm{~N}, 25.15 \\ \text { Found: C, } 43.38 & \mathrm{H}, 3.01 & \mathrm{~N}, 25.25\end{array}$

9) 2-Methylthiazolo $[3,2-a]$ pyrimidin-4-ium perchlorate (III, $\mathbf{R}, \mathbf{R}_{3}=\mathbf{H}, \mathbf{R}_{2}=\mathrm{M}$ e)

Method B : -(with PPA)-

A mixture of 2-amino-5-methyl-1, 3, 4-thiadiazole (3.4 g), 1, 1, 3, 3-tetraethoxypropane (5.5 g), and PPA (20 g) was heated at 140-150" for $60 \mathrm{~min}$. The cooled mixture was diluted with water, and treated with perchloric acid (4.5 g of $70 \%$ $\mathrm{HClO}_{4}$ ). The resulting crystals were recrystallized from DMF-EtOH as yellow needles $(2.6 \mathrm{~g}), \mathrm{m} . \mathrm{p} .213^{\circ}$,

$\begin{array}{rrr}\text { Anal. Calcd. for } \mathrm{C}_{6} \mathrm{H}_{6} \mathrm{O}_{4} \mathrm{~N}_{3} \mathrm{SCl}: \mathrm{C}, 28.64 & \mathrm{H}, 2.35 & \mathrm{~N}, 16.56 \\ \text { Found : C, } 28.67 & \mathrm{H}, 2.76 & \mathrm{~N}, 16.62\end{array}$ 
Method F: -(with conc. $\mathrm{HCl})-$

To a suspension of 2-amino-5-methyl-1,3,4-thiadiazole (3.0 g) in EtOH, conc. $\mathrm{HCl}(5 \mathrm{ml})$ was added dropwise until become a clear solution, then 1, 1, 3, 3tetraethoxypropane $(4.4 \mathrm{~g})$ was added. The solution was heated under reflux for $60 \mathrm{~min}$. After cooling, perchloric acid (25 g of $70 \% \mathrm{HClO}_{1}$ ) was added, and resulting crystals were collected, washed with water, and recrystallized from aq. MeOH as yellow needles. Yield, $54 \%$.
Anal. Calcd. for $\mathrm{C}_{6} \mathrm{H}_{6} \mathrm{O}_{4} \mathrm{~N}_{3} \mathrm{SCl}$ : C, 28.64
H, 2.35
$\mathrm{N}, 16.56$
Found: C, 28.55
$\mathrm{H}, 2.43$
$\mathrm{N}, 16.76$

10) 2, 5, 7-Trimethylthiadiazolo [3,2-a] pyrimidin-4-ium perchlorate, (III, $\mathbf{R}_{1}, \mathbf{R}_{2}$, $\left.\mathbf{R}_{3}=\mathrm{Me}\right)$

A mixture of 2-amino-5-methyl-1, 3, 4-thiadiazole (2.3 g), acetylacetone (2.2 g) and PPA (15 g) was heated at $120-125^{\circ}$ for $90 \mathrm{~min}$. The cooled mixture was diluted with water, and mixed with perchloric acid $(3 \mathrm{~g}$ of $70 \%)$. The resulting crystals were recrystallized from aq. $\mathrm{MeOH}$ as colorless needles $(3.5 \mathrm{~g}), \mathrm{m}$. p. 209".

$\begin{array}{rrr}\text { Anal. Calcd. for } \mathrm{C}_{8} \mathrm{H}_{10} \mathrm{O}_{4} \mathrm{~N}_{3} \mathrm{SCl}: \mathrm{C}, 34.36 & \mathrm{H}, 3.60 & \mathrm{~N}, 15.03 \\ \text { Found : C, 34.41 } & \mathrm{H}, 3.70 & \mathrm{~N}, 14.98\end{array}$

\section{REFERENCES}

Allen, C. F. II., H. R. Beilfuss, D. M. Burness, G. A. Reynolds, J. F. Tinker and J. A. VanAllan 1959 The structure of certain polyazaindenes. J. Org. Chem., 24, 779

Boden, K., T. H. Brown 1971 The reaction of 2 -aminopyridine with some $\beta$-keto-esters in the presence of polyphosphoric acid ethylester. J. Chem. Soc. (C), 2163

Chubb, F. L. and J. Nissenbaum 1959 Hypoglycemic thiadiazoles. Can. J.Chem., 37, 1121: Chem. Abst., 54, 12118b (1960)

Dunwell, D. W. and D. Evans 1971 The reaction of 2-aminothiazoles and 2-aminobenzothiazoles with propiolic acid and its esters. J. Chem. Soc.(C), 2094

Kuderna, J. G., R. S. Skiles and K. Pilgram 1971 Bridgehead nitrogen heterocycles, I. The $2 \mathrm{H}$ ( a nd 4H)-pyrimido[1,2-b]pyridazin-2 (and 4)-one, 3H-Imidazo[1,2-b]pyridazin-2one, and $7 H-1,3,4-$ Thiadiazolo[3,2-a]pyrimidin-7-one systems. J. Org. Chem., 36, 3506

Levin, Ya. A., N. A. Shvinsk and V. A, Kukhtin 1964 Condensed heterocycles. IX, Condensation products of ethyl ethoxymethylenmalonic ester with 2-aminothiazoles and 2aminothiadiazoles. Izv. Akad, Nauk SSSR, Ser. Khim. 1481 : Chem. Abst., 64, 19595f (1966)

Pollak, A., B. Stanovnik and M. Tišler 1971 Pyridazines XXXVII. Pyrimido[1,2-b]pyridazines. J. Org. Chem., 36, 2457

Singh, A., II. Singh, C. K. Dewan and K. S. Narang 1970 Studies on Bisthiazolo[3,2-a: $\left.\Lambda^{\prime}, \overline{5}^{\prime}-d\right]$ pyrimidine derivative. J. Chem. Soc., $(C), 1114$ 